\title{
Macrophages Recruitment and Activation by $\alpha$-gal Nanoparticles Accelerate Regeneration and Can Improve Biomaterials Efficacy in Tissue Engineering
}

\author{
Uri Galili*
}

Department of Surgery, University of Massachusetts Medical School, Worcester, MA 01655, USA

\begin{abstract}
This review describes a novel method for accelerating tissue regeneration by $\alpha$-gal nanoparticles and proposes methods for $\alpha$-gal nanoparticles mediated increased efficacy of biomaterials used in tissue engineering. $\alpha$-Gal nanoparticles present multiple $\alpha$-gal epitopes (Gal $\alpha 1-3 \mathrm{Gal} \beta 1-4 \mathrm{GlcNAc}-\mathrm{R})$ that bind the most abundant natural antibody in all humans- the anti-Gal antibody, constituting $\sim 1 \%$ of immunoglobulins. Anti-Gal/ $\alpha$-gal nanoparticles interaction generates chemotactic complement cleavage peptides that induce rapid and extensive recruitment of macrophages. The subsequent interaction between the Fc portion of anti-Gal coating $\alpha$-gal nanoparticles and Fc $\gamma$ receptors on macrophages activates these cells to produce cytokines/growth factors that promote tissue regeneration and recruit stem cells. Intradermal injection of $\alpha$-gal nanoparticles induces localized extensive recruitment and activation of macrophages. These macrophages disappear within 3 weeks without altering normal skin architecture. Application of $\alpha$-gal nanoparticles onto wounds of anti-Gal producing animals reduces healing time by $40-70 \%$. $\alpha$-Gal nanoparticles injected into ischemic myocardium induce extensive recruitment of macrophages that secrete cytokines preserving the structure of the ischemic tissue. These macrophages may recruit progenitor cells and/or stem cells that are guided by myocardial microenvironment and extracellular matrix to differentiate into cardiomyocytes. $\alpha$-Gal nanoparticles applied to nerve injures will recruit macrophages that can promote angiogenesis required for induction of axonal sprouting and thus may regenerate the severed nerve. In tissue engineering, incorporation of $\alpha$-gal nanoparticles into decellularized tissue and organ implants may improve in vivo regeneration and restore biological function of implants because of accelerated recruitment of macrophages and stem cells.
\end{abstract}

Keywords: Skin injury, wound healing, macrophage activation, $\alpha$-gal nanoparticles, anti-Gal antibody, myocardium regeneration, nerve regeneration, biomaterials, tissue engineering.

This review describes a novel method for recruitment and activation of macrophages within injured tissues by $\alpha$-gal nanoparticles. The review further proposes the use of these nanoparticles in biomaterials for tissue engineering, in order to accelerate and increase the efficacy of tissue repair and regeneration processes. The method is based on harnessing the immunological potential of the natural anti-Gal antibody, which is the most abundant natural antibody in humans. Anti-Gal interacts specifically with a carbohydrate antigen called "the $\alpha$-gal epitope" with the structure Gal $\alpha 1-3 \mathrm{Gal} \beta 1$ 4GlcNAc-R. Nanoparticles presenting multiple $\alpha$-gal epitopes and called $\alpha$-gal nanoparticles introduce these epitopes into injury sites or into biomaterials. The interaction of antiGal with $\alpha$-gal epitopes on $\alpha$-gal nanoparticles activates the complement system for rapid chemotactic recruitment of macrophages. This interaction further induces pro-healing functions in the recruited macrophages which may recruit stem cells. The review describes studies on the efficacy of anti-Gal/ $\alpha$-gal nanoparticles interaction in acceleration of

*Address correspondence to this author at the Department of Surgery, HB774, University of Massachusetts Medical School, 55 Lake Avenue North, Worcester, MA 01655, USA; Tel: 508-856-4188;

Fax: 508-856-4106; E-mail: Uri.Galili@umassmed.edu wound healing and in recruitment and activation of macrophages. The review further proposes possible uses of $\alpha$-gal nanoparticles in facilitating regeneration of tissues affected by internal injures such as ischemia of the myocardium. In addition, the review suggests methods for incorporating these nanoparticles into biomaterials used for tissue engineering. The ultimate purpose of this review is to interest researchers in the area of tissue engineering, repair and regeneration in studying the possible uses of $\alpha$-gal nanoparticles in various regenerative treatments for increasing the efficacy of such treatments and for accelerating the regenerative process.

\section{IMMUNE RECRUITMENT OF MACROPHAGES FOR TISSUE HEALING AND REGENERATION}

Macrophages are the pivotal cells in early stages of injury healing and tissue regeneration. Macrophages migrating into injury sites debride the injured tissue by phagocytosis. Subsequently, upon transition into the pro-healing phase, macrophages orchestrate regeneration by secreting a variety of cytokines/growth factors that induce angiogenesis and regeneration of the injured tissue $[1,2]$. Macrophages are recruited into wounds within several days post injury by cytokines such as MIP-1 and MCP-1 released from cells within 
and around injury sites $[3,4]$. This recruitment can be markedly accelerated by antibodies interacting with various antigens and causing local activation of the complement system. Complement activation results in generation of complement cleavage peptides such as $\mathrm{C} 5 \mathrm{a}$ and $\mathrm{C} 3 \mathrm{a}$ which are chemotactic factors that induce rapid extravasation of monocytes, and their differentiation into macrophages which migrate along the chemotactic gradient $[5,6]$. This immune mediated recruitment of macrophages and their activation can be achieved in humans by harnessing the natural anti-Gal antibody. The harnessing of this antibody is feasible by using $\alpha$ gal nanoparticles.

THE NATURAL ANTI-GAL ANTIBODY, $\alpha$-GAL EPITOPES AND $\alpha$-GAL NANOPARTICLES

Anti-Gal is the most abundant natural antibody in all humans, constituting $\sim 1 \%$ of circulating immunoglobulins in young [7] and in elderly individuals [8]. Anti-Gal binds specifically to a carbohydrate antigen called the $\alpha$-gal epitope with the structure Gal $\alpha 1-3 \mathrm{Gal} \beta 1-4 \mathrm{GlcNAc}-\mathrm{R}$ [9]. This antibody is produced throughout life in response to continuous antigenic stimulation by bacteria of the normal gastrointestinal flora [10]. Anti-Gal is naturally produced also in Old World monkeys (monkeys of Asia and Africa) and in apes, however, it is absent in other mammals [11]. In contrast, other mammalian species including nonprimate mammals (e.g. mice, rats, rabbits, dogs, pigs, etc.) as well as prosimians and New World monkeys (monkeys of South America) lack the anti-Gal antibody but all produce its ligand the $\alpha$-gal epitope, by using a glycosylation enzyme called $\alpha 1,3$ galactosyltransferae $(\alpha 1,3 \mathrm{GT})[11,12]$. The $\alpha 1,3 \mathrm{GT}$ gene was inactivated in ancestral Old World monkeys and apes 20-28 million years ago, resulting in elimination of $\alpha$-gal epitope synthesis in these primates and the appearance of the natural anti-Gal antibody [13-17].

The activity of anti-Gal can be manipulated in humans by the use of $\alpha$-gal nanoparticles. These are submicroscopic $\alpha$ gal liposomes comprised of glycolipids with multiple $\alpha$-gal epitopes ( $\alpha$-gal glycolipids), phospholipids and cholesterol [18, 19] (Fig. 1). Since $\alpha$-gal glycolipids comprise most of the glycolipids in rabbit red blood cell (RBC) membranes and since these cell membranes are the richest source of $\alpha$ gal glycolipids in mammals [20-23], rabbit RBC are a convenient natural source for preparation of $\alpha$-gal liposomes and $\alpha$-gal nanoparticles $[18,19,24]$. For this purpose, glycolipids, phospholipids and cholesterol are extracted from rabbit RBC membranes in a solution of chloroform and methanol [25]. The dried extract is sonicated in saline, using a sonication bath, to generate liposomes (size of $1-10 \mu \mathrm{m}$ ) that present multiple $\alpha$-gal epitopes. These liposomes (referred to as $\alpha$-gal liposomes) are further sonicated into submicroscopic particles called $\alpha$-gal nanoparticles, by the use of a sonication probe. $\alpha$-Gal nanoparticles have the same composition as $\alpha$-gal liposomes, however their size range is $10-300 \mathrm{~nm}$. The $\alpha$-gal nanoparticles suspension is further sterilized by filtration through a $0.2 \mu \mathrm{m}$ filter. It is probable that synthetic $\alpha$-gal nanoparticles can be generated, as well, by currently available technologies.

A schematic presentation of an $\alpha$-gal nanoparticle is illustrated in Fig. (1A). This nanoparticle has a wall of phos- pholipids and cholesterol in which $\alpha$-gal glycolipids are anchored via the fatty acid tails of their ceramide portion. The illustrated glycolipid has 10 sugar units in its carbohydrate chain and two branches (antennae), each capped with an $\alpha$ gal epitope. $\alpha$-Gal glycolipids in rabbit RBC membranes are of various lengths ranging from 5 to 40 carbohydrate units carrying 1-8 branches each capped with an $\alpha$-gal epitope [22, $23,25]$. The various components of $\alpha$-gal nanoparticles are illustrated in Fig. (1B) where the nanoparticles are dissolved in chloroform:methanol solution and run on a thin layer chromatography (TLC) plate. With the exception of the glycolipid ceramide tri-hexoside which lacks $\alpha$-gal epitopes (present also in human RBC membranes), all other glycolipids with 5-25 carbohydrate units separated on the plate are capped with $\alpha$-gal epitopes (i.e. are $\alpha$-gal glycolipids) as indicated by immunostaining with a monoclonal anti-Gal antibody.

The number of $\alpha$-gal epitopes on $\alpha$-gal nanoparticles is very high, corresponding to $\sim 10^{15} \alpha$-gal epitopes per $\mathrm{mg} \alpha$ gal nanoparticles [18]. From 1 liter of rabbit RBC it is possible to prepare 3-4 grams of $\alpha$-gal nanoparticles. These $\alpha$ nanoparticles are highly stable since they contain no tertiary structures. Accordingly, no changes in expression of $\alpha$-gal epitopes have been found in $\alpha$-gal nanoparticles kept at $4^{\circ} \mathrm{C}$ for 4 years, in comparison with freshly produced $\alpha$-gal nanoparticles (unpublished observations).

It should be stressed that the study of anti-Gal mediated acceleration of wound healing by $\alpha$-gal nanoparticles cannot be performed in standard experimental animal models such as mice, rats, guinea-pigs, rabbits and pigs. All these mammals produce $\alpha$-gal epitopes on their cells and thus cannot produce the anti-Gal antibody $[11,12]$. The only two nonprimate experimental animal models which are suitable for anti-Gal studies are $\alpha 1,3 \mathrm{GT}$ knockout mice (GT-KO mice) produced in the mid-1990s [26, 27] and $\alpha 1,3 \mathrm{GT}$ knockout pigs (GT-KO pigs) produced in the last decade [28-30]. These two knockout animal models lack $\alpha$-gal epitopes and can produce the anti-Gal antibody. Old World monkeys, which naturally produce the anti-Gal antibody also can serve as animal models for evaluating therapies associated with the natural anti-Gal antibody.

\section{ANTI-GAL/ $\alpha$-GAL NANOPARTICLES INTERAC- TION INDUCES RAPID AND EXTENSIVE MACRO- PHAGE RECRUITMENT}

Interaction between serum anti-Gal and $\alpha$-gal epitopes results in activation of the complement system. Transplantation of pig xenografts in monkeys is a dramatic demonstration of this complement activation process. Binding of circulating anti-Gal to the multiple $\alpha$-gal epitopes on pig endothelial cells lining the blood vessels of pig kidney or heart xenografts [31, 32], or to New World monkey heart xenograft [33], results in activation of the complement system. The activated complement cascade causes lysis of the endothelial cells, collapse of the vascular bed and hyperacute rejection of the xenograft within 30 minutes to several hours [31-33]. A similar activation of the complement system occurs when serum anti-Gal binds to the multiple $\alpha$-gal epitopes on $\alpha$-gal nanoparticles. This complement activation results in the generation of chemotactic complement cleav- 

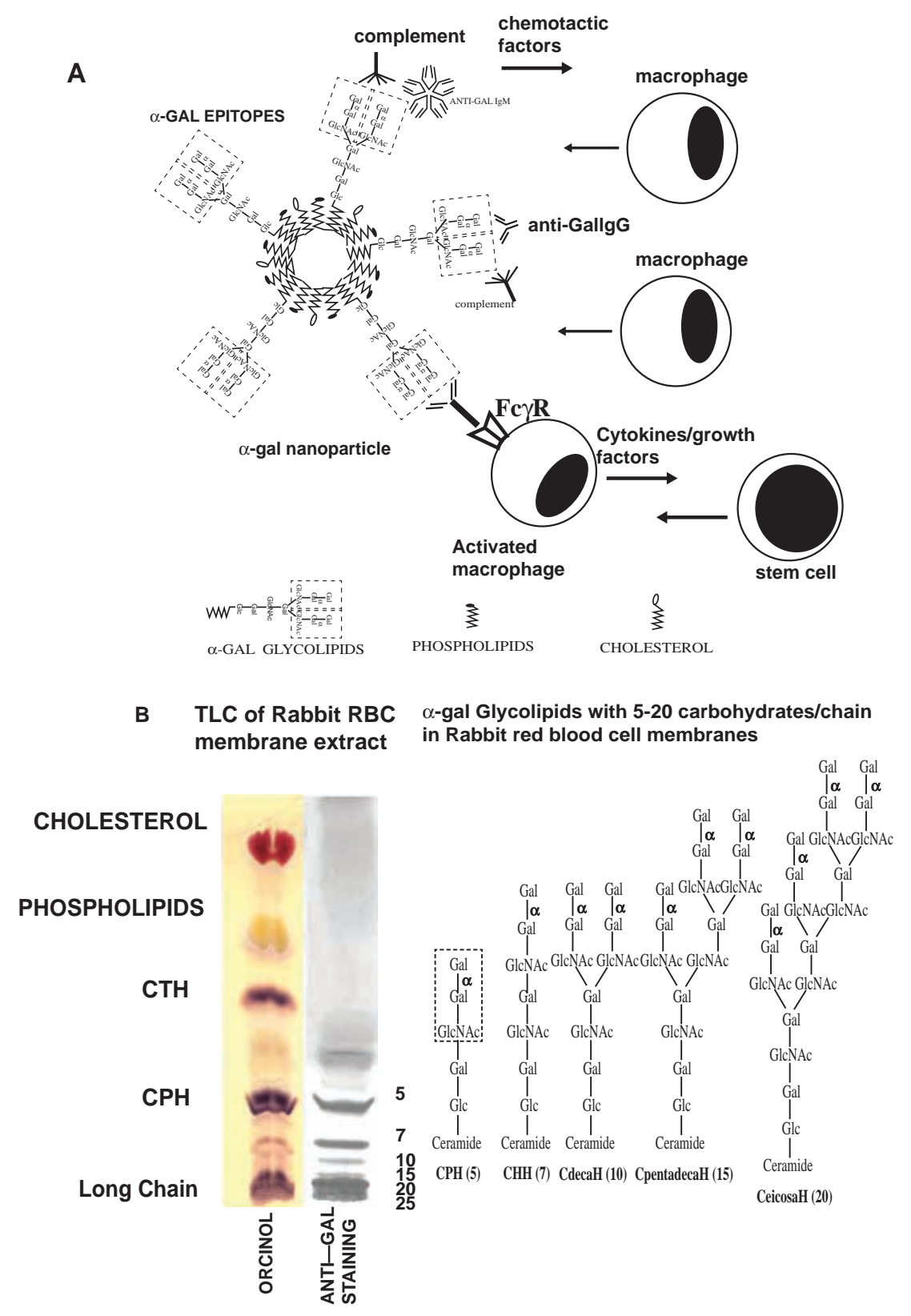

Fig. (1). Structure of $\alpha$-gal nanoparticles and their suggested functions in wounds. A. Schematic illustration of an $\alpha$-gal nanoparticle with $\alpha$-gal glycolipids containing 10 carbohydrates in two branches, each capped with an $\alpha$-gal epitope (marked by a dashed line rectangle). When $\alpha$-gal nanoparticles are applied to wounds, binding of the natural anti-Gal antibody to their $\alpha$-gal epitopes results in activation of the complement system. The produced chemotactic complement cleavage peptides induce rapid recruitment of macrophages. Anti-Gal coating $\alpha$-gal nanoparticles further interacts via its Fc "tail" with $\mathrm{Fc} \gamma$ receptors $(\mathrm{Fc} \gamma \mathrm{R})$ on macrophages. This interaction activates macrophages to produce and secrete a variety of pro-healing cytokines/growth factors which affect tissue repair and recruit stem cells. The components of the $\alpha$-gal nanoparticles are illustrated at the bottom of the figure (modified from ref. 19). B. Characterization of $\alpha$-gal nanoparticle components extracted from rabbit RBC membranes, separated on thin layer chromatography (TLC) plate and stained nonspecifically by orcinol (left lane) or immunostained with anti-Gal antibody (right lane). The glycolipids, phospholipids and cholesterol are stained by orcinol in the left lane. CTH- ceramide trihexoside which lacks $\alpha$-gal epitopes is also found human RBC. Ceramide pentahexoside (CPH with 5 carbohydrates), and larger glycolipids are indicated as bands beneath $\mathrm{CPH}$ (left lane). All glycolipids with $\geq=5$ sugar units in their carbohydrate chains have $\alpha$ gal epitopes and thus are immunostained by anti-Gal in the right lane. Their size (number of carbohydrate units) is indicated right of the lane. The structure of these $\alpha$-gal glycolipids with 5, 7, 10, 15 and 20 carbohydrates is illustrated as well in the right panel (modified from ref. 25).

age peptides that are among the most potent physiologic chemotactic factors. These include C5a and C3a complement cleavage peptides which induce rapid migration of macro- phages into the site of $\alpha$-gal nanoparticles application (Fig. 1A) [18]. 
In studies with $\alpha$-gal nanoparticles injected intradermal in anti-Gal producing GT-KO mice, macrophages were found to be recruited by this chemotactic mechanism. The macrophages reached the injection site within $24 \mathrm{~h}$ and continued migrating into that site for several days (Fig. 2) [18]. Identification of the migrating cells as macrophages could be established by immunostaining with the macrophage specific anti-4/F80 antibody [18]. The macrophages were found at the injection site for 14-17 days. These macrophages completely disappeared after 21 days without changing skin architecture. No chronic granulomas and no detrimental inflammatory responses were found in such $\alpha$-gal nanoparticles injection sites. The in vivo recruitment of macrophages by $\alpha$-gal nanoparticles was further validated by subcutaneous implantation of biologically inert sponge discs (made of polyvinyl alcohol- PVA) that contained $10 \mathrm{mg} \alpha$-gal liposomes (i.e. nanoparticles prior to sonication into submicroscopic liposomes). After 6 days, the implanted PVA sponge discs contained multiple migrating cells of which $>99 \%$ were immunostained by the macrophage specific antiCD11b antibody, whereas no T cells or B cells were detected [24]. Sponges lacking $\alpha$-gal liposomes contained 10 fold less macrophages than those containing $\alpha$-gal liposomes. Similarly, sponges containing $\alpha$-gal liposomes and implanted into wild type mice (i.e. mice not producing anti-Gal) were devoid of infiltrating macrophages [24]. Another example for the extensive recruitment of macrophages by $\alpha$-gal nanopar- ticles is described below in ischemic myocardium of GT-KO mice injected with these nanoparticles.

\section{ACTIVATION OF MACROPHAGES BY ANTI-GAL COATED $\alpha$-GAL NANOPARTICLES}

After the recruited macrophages reach the $\alpha$-gal nanoparticles, the Fc "tails" of anti-Gal coating these nanoparticles bind to $F c \gamma$ receptors $(F c \gamma R)$ on the macrophages (Fig. 1A). This extensive binding to Fc $\gamma \mathrm{R}$ on macrophages is visualized in Fig. (3) where anti-Gal coated $\alpha$-gal nanoparticles were incubated in vitro with cultured macrophages of $\alpha 1,3 \mathrm{GT}$ knockout pig origin (GT-KO pig). Multiple $\alpha$-gal nanoparticles attach to the macrophages via the $\mathrm{Fc} / \mathrm{Fc} \gamma \mathrm{R}$ interaction. In the absence of anti-Gal, no significant binding of $\alpha$-gal nanoparticles to macrophages was observed. This Fc/Fc $\gamma \mathrm{R}$ interaction generates a trans-membrane signal that activates the macrophages to produce various cytokines/growth factors that promote tissue repair and regeneration. Analysis of growth factor secretion into the culture medium demonstrated increased production of VEGF, whereas quantitative PCR demonstrated increased production of FGF, IL1, PDGF and CSF [18]. Based on these observations it was hypothesized that the high concentration of multiple cytokines/growth factors secreted in vivo by activated macrophages following this $\mathrm{Fc} / \mathrm{Fc} \gamma \mathrm{R}$ interaction is likely to mediate wound healing and tissue regeneration [18].
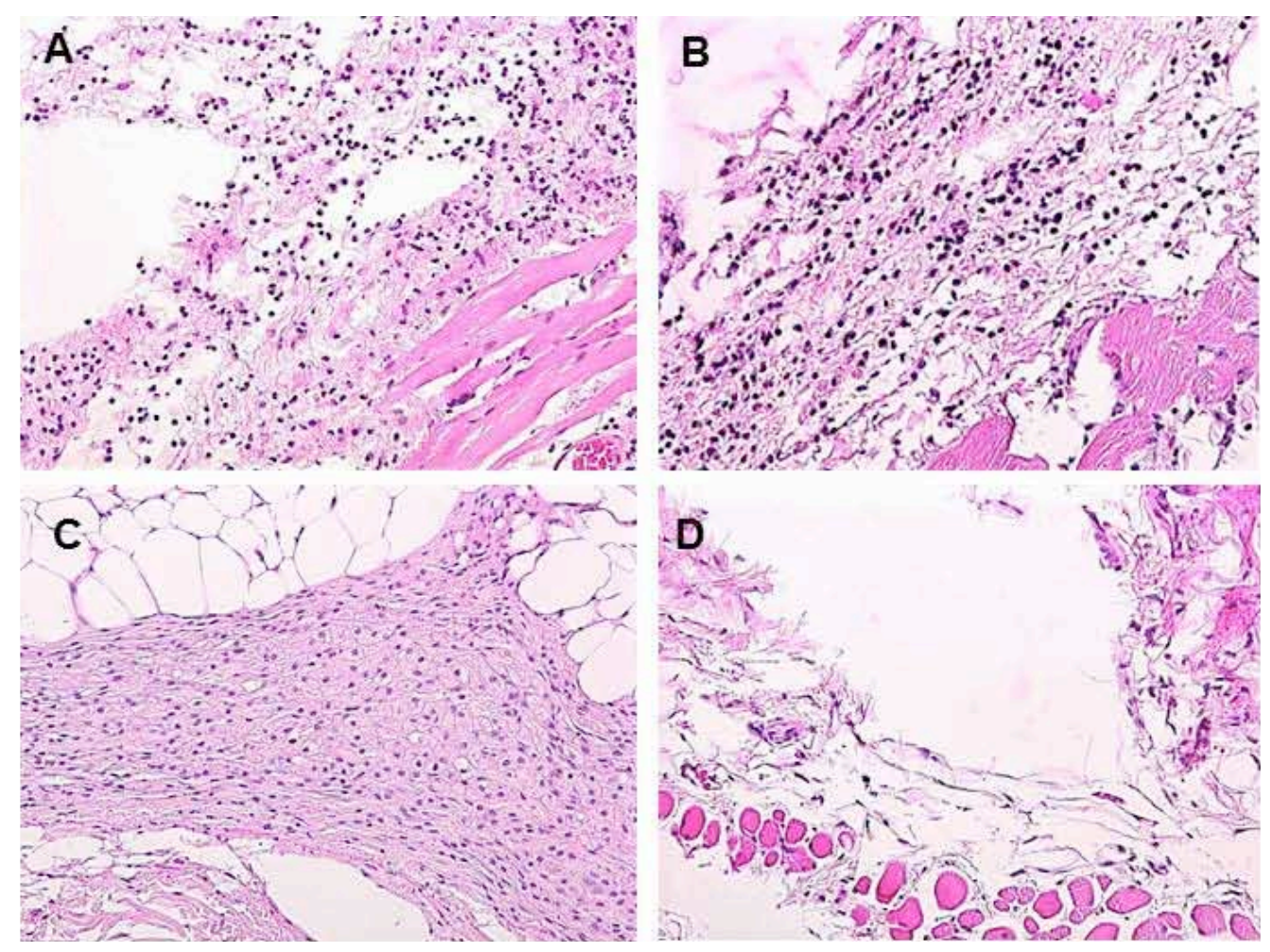

Fig. (2). Rapid recruitment of macrophages by $\alpha$-gal nanoparticles injected into the skin of GT-KO mouse. (A) The injection site of $1 \mathrm{mg} \alpha$-gal nanoparticles in $0.1 \mathrm{ml}$ suspension, inspected after $24 \mathrm{~h}$. Macrophages are recruited around the injection site. The injection site is the empty area in the dermis as the $\alpha$-gal nanoparticles are dissolved and washed away by the alcohol used in the hematoxylin \& eosin (H\&E) staining process (x100). (B) The injection site after $48 \mathrm{~h}$ demonstrating increased recruitment of macrophages (x100). (C) The injection area after 6 days showing multiple activated macrophages bordering each other and displaying ample cytoplasm due to the activation process (x200). (D) Control injection sites injected only with saline and inspected after $48 \mathrm{~h}$. The injection sites display no recruitment of macrophages (x100). Representative specimens of 5 mice with similar results. 

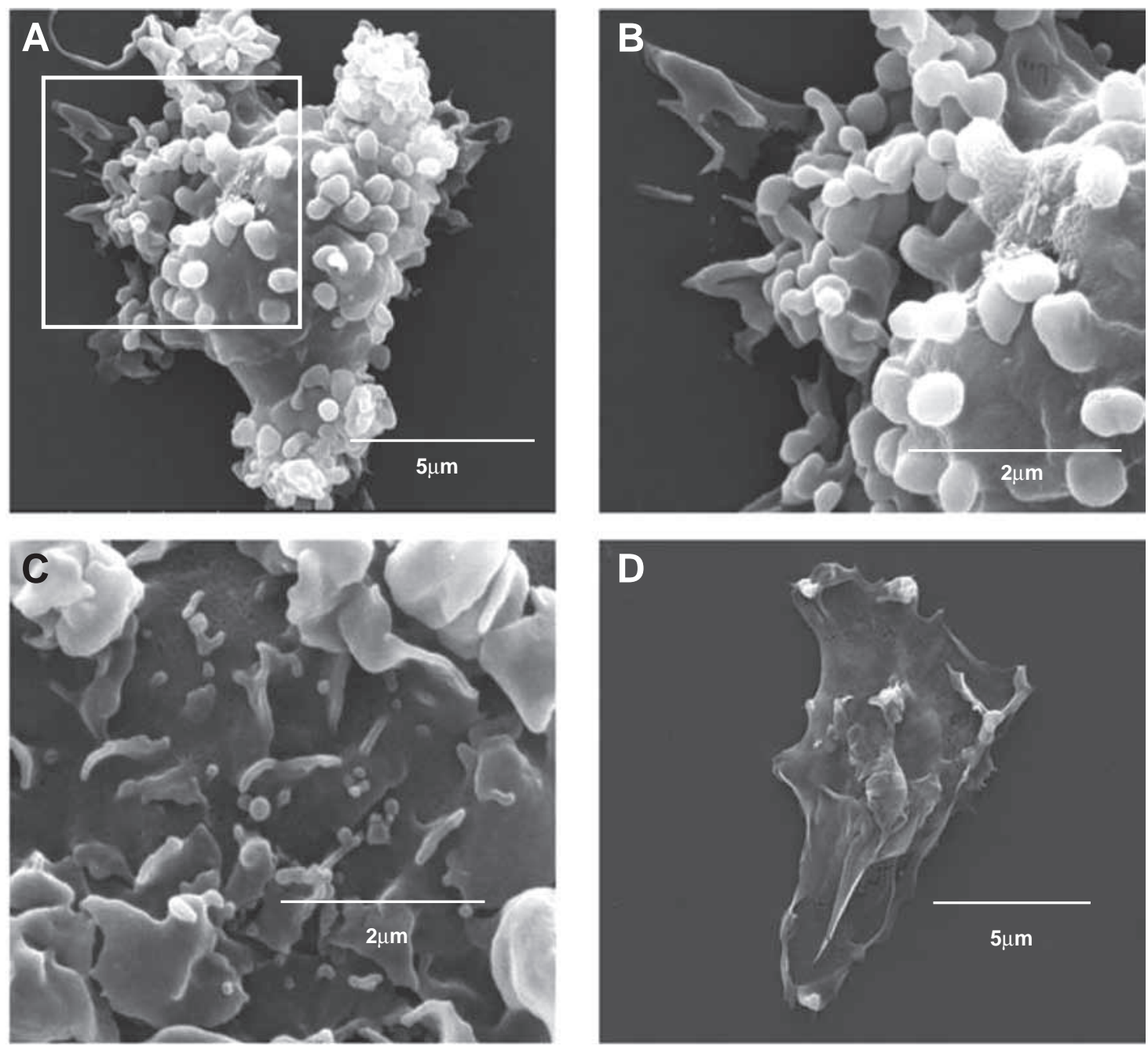

Fig. (3). Binding of anti-Gal coated $\alpha$-gal nanoparticles to macrophages, as evaluated by scanning electron microscopy. A-C. The $\alpha$ gal nanoparticles coated with natural GT-KO pig anti-Gal antibody were incubated with adherent GT-KO pig macrophages for $2 \mathrm{~h}$ at room temp. The macrophages were then extensively washed to remove nonadherent nanoparticles and subjected to scanning electron microscopy processing and analysis. The surface of a representative macrophage is covered with $\alpha$-gal nanoparticles as a result of Fc/Fc $\gamma \mathrm{R}$ interaction. The inset in (A) is enlarged in (B). In A and B the size of the $\alpha$-gal nanoparticles is $\sim 100-300 \mathrm{~nm}$. In (C) the size is 10-30nm. D. A macrophages incubated with $\alpha$-gal nanoparticles that were not coated with anti-Gal antibody. No nanoparticles binding is observed.

\section{ACCELERATING WOUND AND BURN HEALING IN GT-KO MICE}

The potential of $\alpha$-gal nanoparticles for accelerating healing and regeneration of injured tissues was demonstrated in studies on wound and burn healing. Excisional deep skin wounds were formed in anti-Gal producing GT-KO mice. Wounds were treated with spot bandage coated with $10 \mathrm{mg}$ $\alpha$-gal nanoparticles, nanoparticles lacking $\alpha$-gal epitopes (from GT-KO pig RBC), or with saline. Treatment of wounds with $\alpha$-gal nanoparticles resulted in $95-100 \%$ healing (i.e. regeneration of epidermis) by day 6 post injury, whereas wounds treated with nanoparticles that lack $\alpha$-gal epitopes or with saline displayed $<20 \%$ healing at that time [18]. Measurements of wound healing at various time points indicated that treatment with $\alpha$-gal nanoparticles reduced the healing time by $\sim 70 \%$ in comparison to saline control. Histological evaluation of wounds further indicated that the processes of vascularization, fibroblast migration and colla- gen deposition in the dermis also were accelerated in wounds treated with $\alpha$-gal nanoparticles in comparison to saline treated wounds [18].

Anti-Gal mediated accelerated healing was also demonstrated in GT-KO mice with burn injuries treated with $10 \mathrm{mg}$ $\alpha$-gal liposomes ( $\alpha$-gal nanoparticles prior to sonication) [24]. The healing of $\alpha$-gal liposomes treated burns was twice as fast as that of saline treated burns. Histology of the healing burns demonstrated accelerated migration of neutrophils followed by macrophages migration into treated burns. This rapid migration is further followed by accelerated regeneration of the epidermis and deposition of collagen by recruited fibroblasts in the regenerating dermis of the burns. Similar studies in wild-type mice synthesizing autologous $\alpha$-gal epitopes and lacking anti-Gal antibody demonstrated no acceleration in healing following $\alpha$-gal liposomes treatment [24], further indicating that the observed acceleration in the healing process is associated with anti-Gal/ $\alpha$-gal epitope interaction. 


\section{ACCELERATED WOUND HEALING DECREASES SCAR FORMATION IN GT-KO MICE}

Scar formation due to fibrosis of injured tissues is the default mechanism for repair and regeneration of both external and internal injuries. In the absence of recruited stem cells that differentiate in order to restore the original cell composition of the injured tissue, fibroblasts migrate into the injury site, secrete collagen and form a scar. Indeed, saline treated wounds in GT-KO mice that were inspected after 28 days displayed wide areas of dense fibrotic dermis devoid of skin appendages, and epidermal hyperplasia, both characteristic to scar formation [18]. In contrast, epidermis in $\alpha$-gal nanoparticles treated wounds displayed normal thickness and the collagen in the dermis displayed normal density. These healed wounds also contained regenerating skin appendages such as hair follicles and sebaceous glands, as well as fat cells and muscle cells, however, no granulomas were detected [18].

The lack of scar tissue in the $\alpha$-gal nanoparticles treated wounds strongly suggests that the rapid anti-Gal mediated recruitment and activation of macrophages results in localized secretion of cytokines/growth factors that promote tissue healing, recruitment of stem cells and restoration of the cellular components of normal skin. The rapid regeneration of the normal skin components prevents the induction of the default fibrosis and scar formation processes. It remains to be determined whether this mechanism of increased efficacy of wound healing by $\alpha$-gal nanoparticles is applicable to wound treatment in humans. If successful, this treatment may facilitate accelerated wound healing in the elderly as well, since natural anti-Gal antibody production is observed also in elderly individuals above the age of 75 [8].

\section{ACCELERATING WOUND HEALING BY $\alpha$-GAL NANOPARTICLES IN GT-KO PIGS}

The acceleration of wound healing by $\alpha$-gal nanoparticles treatment was validated in the large animal model of GT-KO pigs [19]. These pigs lack $\alpha$-gal epitopes because of targeted disruption (knockout) of the $\alpha 1,3 \mathrm{GT}$ gene [28-30] and thus, they produce the natural anti-Gal antibody. The study of wound healing in pigs is of further interest since their skin structure is similar to that of human skin. The natural antiGal antibody in GT-KO pig sera readily binds to $\alpha$-gal nanoparticles and activates pig complement, as well as anti$\mathrm{Gal}$ in human serum. Excisional 20x20mm square wounds ( $3 \mathrm{~mm}$ deep) were formed on the back of 3 month old GTKO pigs. Borders of the wounds were marked by tattooed dots prior to wounding. The wounds were covered with dressing coated with $100 \mathrm{mg} \alpha$-gal nanoparticles, or with saline as control. On day 7, all wounds were filled with granulation tissue. Although on day 7 there was no significant difference in the size of wounds treated with $\alpha$-gal nanoparticles in comparison to saline treated wounds, the former wounds contained many more macrophages [19].

On day 13 , the wounds treated with $\alpha$-gal nanoparticles were completely or almost completely covered with regenerating epidermis (Fig. 4), whereas complete healing of saline treated wounds was observed only 18-22 days post wounding. On average, wounds treated with $\alpha$-gal nanoparticles were 10 fold smaller than saline treated wounds and the extent of angiogenesis in the former was much higher [19]. No significant differences were observed, however, in wound contraction (marked by conversion of tattooed dots into stretched lines) between $\alpha$-gal nanoparticles treated wounds and saline treated wounds (Fig. 4). Interestingly, the regenerating dermis in $\alpha$-gal nanoparticles treated wounds allowed

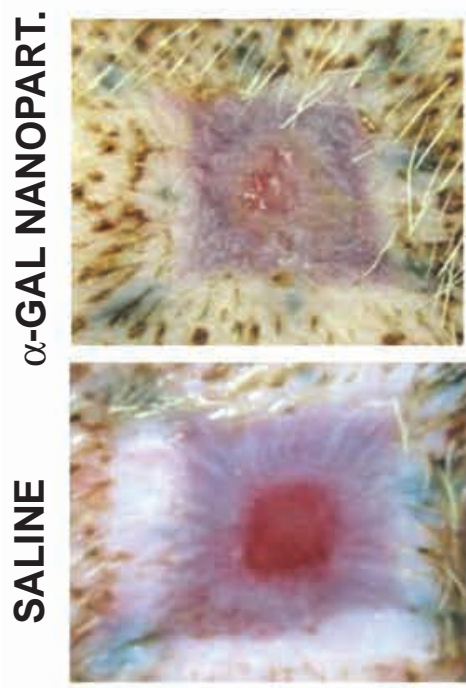

Pig 01



Pig 04

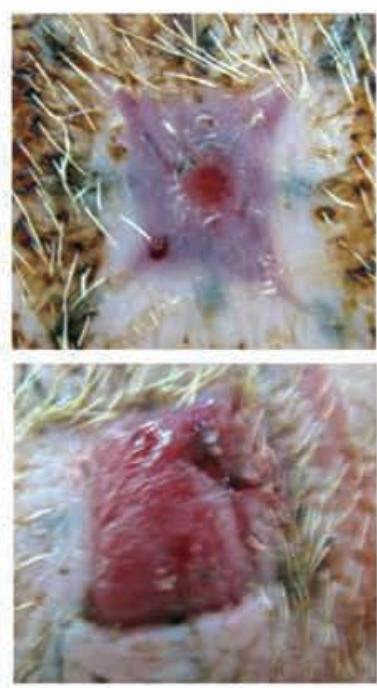

Pig 05



Pig 09

Fig. (4). Day 13 morphology of excisional wounds $(20 x 20 \mathrm{~mm}, \sim 3 \mathrm{~mm}$ deep) in the skin of GT-KO pigs treated by $\alpha$-gal nanoparticles or saline. Each pair of wounds is of the same pig. In most pigs, the surface of wounds treated with 100mg $\alpha$-gal nanoparticles is completely covered by regenerating epidermis whereas epidermis regeneration is significantly slower in saline treated wounds. Results are representative of $8 \mathrm{KO}$ pigs studied (from ref. 19). 

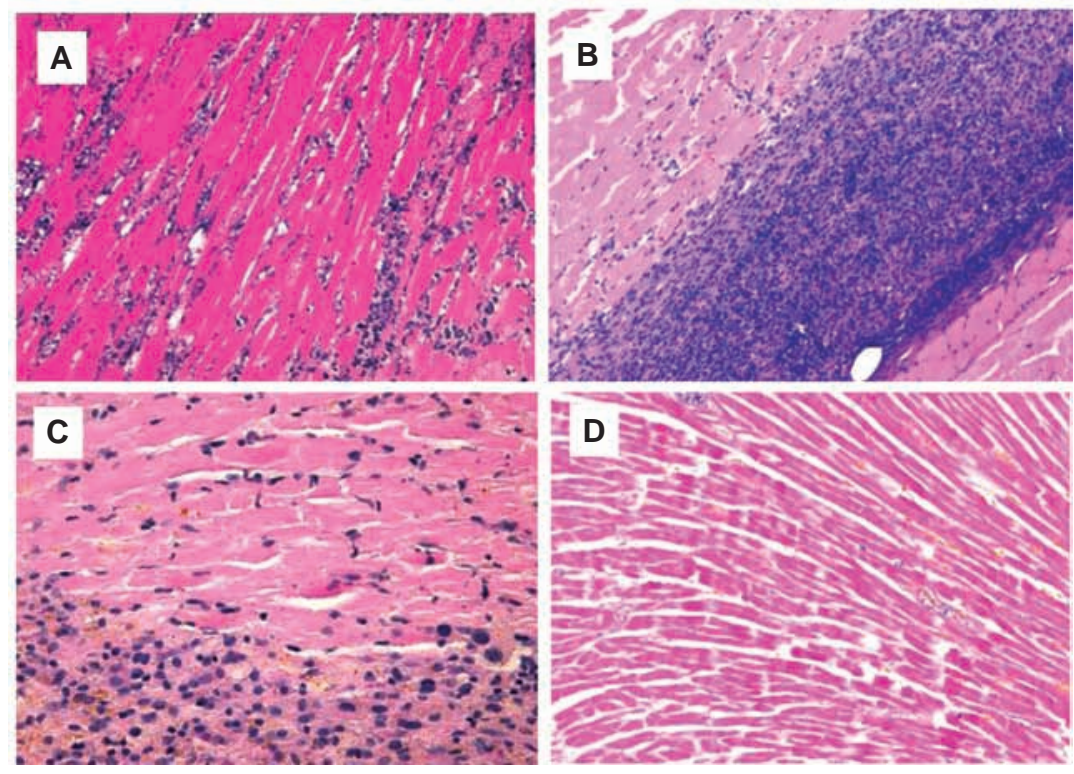

Fig. (5). Recruitment of macrophages into ischemic heart tissue by $\boldsymbol{\alpha}$-gal nanoparticles- The myocardium of GT-KO mouse hearts was injected with $1 \mathrm{mg} \alpha$-gal nanoparticles (B-D) or with saline (A) prior to subcutaneous implantation. The implanted hearts were harvested after 2 weeks $(\mathbf{A}, \mathbf{B})$ or 4 weeks $(\mathbf{C , D})$ and subjected to H\&E staining and histological analysis. A. Hearts injected with saline displayed necrosis and infiltration of neutrophils that are characteristic to ischemic tissues (x100). B. Hearts injected with $\alpha$-gal nanoparticles displayed extensive infiltration of macrophages at the injection site (x100). C. The implanted heart harvested 4 weeks post $\alpha$-gal nanoparticle injection and implantation. Macrophages migrated from injected area (lower area) into non-injected area (x200). D. The heart injected with $\alpha$-gal nanoparticles harvested 4 weeks after implantation. The area of myocardium presented was far from the injection site area and was devoid of infiltrating macrophages. (x100). Note that despite lack of nuclei in ischemic cardiomyocytes, the myocardium was not necrotic and the cardiomyocytes preserved their structure, including the intercalated discs. Displayed hearts are representative of 5 hearts per group [57].

for growth of skin appendages including hair and sebaceous glands. Monitoring the wounds for 60 days indicated that despite the accelerated healing, wounds treated with $\alpha$-gal nanoparticles did not form excessive scars and did not display keloid formation [19]. These findings suggest that similar treatment in humans may induce accelerated wound healing without adverse effects.

In addition to direct application of the $\alpha$-gal nanoparticles on wounds or on bandages, these nanoparticles may be incorporated into sheets or flowable gel made of biodegradable scaffold materials such as natural or recombinant collagen [34]. Treatment of wounds or burns with biodegradable sheets containing $\alpha$-gal nanoparticles may increase their wound healing efficacy by accelerated recruitment and activation of macrophages due to anti-Gal and complement proteins diffusion into such sheets and interaction of this antibody with the $\alpha$-gal nanoparticles, causing complement activation and formation of gradients of complement chemotactic factors.

\section{POSSIBLE BENEFICIAL EFFECTS IN REGENERA- TION OF ISCHEMIC MYOCARDIUM AND OF IN- JURED NERVES}

Two examples of internal injuries in which $\alpha$-gal nanoparticles may have regenerative effects are those of ischemic myocardium and injured nerves.

Ischemia post myocardial infarction- In post myocardial infarction, as in wound healing, macrophages migrate to the injured myocardium, debride it of dead cells and secrete cy- tokines/growth factors that recruit stem cells from the circulation and/or progenitor cells from adjacent uninjured myocardium (referred here collectively as stem/progenitor cells). The recruited stem/progenitor cells receive cues from the adjacent healthy cells, the microenvironment and the extracellular matrix (ECM) to differentiate into cardiomyocytes that regenerate the tissue and restore its physiologic activity [35-38]. Myocardium with limited ischemic damage may display spontaneous regeneration by this mechanism. However, in more extensive ischemic damage, migration of macrophages into injured myocardium and the recruitment of stem/progenitor cells processes are too slow to prevent irreversible fibrosis which is the default mechanism for tissue repair. It is suggested that this fibrosis may be reduced and possibly avoided by direct transendocardial injection of $\alpha$-gal nanoparticles into the injured myocardium. This can be achieved by an injecting catheter within the left ventricle, shortly after the myocardial infarction event, so that regeneration is induced prior to onset of fibrosis. Injected $\alpha$-gal nanoparticles will bind anti-Gal and induce rapid chemotactic migration of macrophages which will be activated to secrete cytokines/growth factors that recruit stem/progenitor cells. These recruited cells may be guided by the microenvironment and the ECM to differentiate into cardiomyocytes that restore the biological activity of the ischemic myocardium.

An example for the high ability of $\alpha$-gal nanoparticles to recruit macrophages into ischemic myocardium is illustrated in Fig. (5). Harvested GT-KO mouse hearts were injected with saline or with $1 \mathrm{mg} \alpha$-gal nanoparticles and implanted 




Fig. (6). Recruitment of macrophages into a plasma clot containing $\alpha$-gal nanoparticles. Clots of human plasma mixed with $\alpha$-gal nanoparticles $(10 \mathrm{mg} / \mathrm{ml})$ were placed on skin wounds of GT-KO mice. A. A representative clot after 3 days. Initial migration of macrophages is observed within the clot and in the dermis bordering it. B A representative clot after 6 days, demonstrating extensive recruitment of macrophages within the clot and growth of epidermis over the clot. The broken lines mark the approximate edge of the clot. Representative specimens from 5 mice in each time point.

subcutaneously in anti-Gal producing GT-KO mice. The implanted hearts were not connected to the recipient's circulation. In saline injected implanted hearts, the process of necrosis was evident in specimens retrieved after two weeks. This necrosis is characterized by the eosinophilic staining of the cardiomyocytes and the multiple neutrophils in the tissue. However, in $\alpha$-gal nanoparticles injected hearts, the interaction between $\alpha$-gal nanoparticles and anti-Gal induced extensive migration of macrophages into the ischemic myocardium within two weeks post implantation. After four weeks, the recruited macrophages migrated into the adjacent non-injected myocardium. The activation of the recruited macrophages by anti-Gal coated $\alpha$-gal nanoparticles resulted in secretion of cytokines that conserved the myocardium structure and delayed necrosis, as observed after four weeks in areas devoid of infiltrating macrophages (Fig. 5). Heart implants injected with saline completely disappeared after four weeks.

Previous observations demonstrated the significance of macrophages in inducing angiogenesis and recruitment of stem cells in post infarct ischemic myocardium [37-39]. In view of these studies, it is possible that the extensive migration and activation of macrophages following intramyocardial injection of $\alpha$-gal nanoparticles may result in effective recruitment of stem/progenitor cells which receive the appropriate guidance from the structurally conserved myocardium in order to differentiate into cardiomyocytes that restore myocardial function.

Regeneration of injured nerves- Activated macrophages are also pivotal in regeneration of injured nerves, as in spinal cord injury. Regeneration of nerves requires regrowth of multiple sprouts from the injured axons. These sprouts "attempt" to reconnect across the lesion and grow into the distal axonal tube of the damaged neurons. This axonal sprout growth occurs along de novo generated blood capillaries in the nerve lesion site and thus depends on local angiogenesis induced by VEGF secreted from pro-healing macrophages recruited to the injury site $[40,41]$. If this growth of axonal sprouts is delayed because of insufficient recruitment of macrophages into the injury site, the ongoing fibrosis will irreversibly prevent regeneration of the injured nerve. The wound healing studies $[18,19]$ suggest that $\alpha$-gal nanoparticles applied to nerve injury sites will bind anti-Gal, induce rapid macrophage migration and activation of these macrophages for the secretion of VEGF. This process is likely to result in local angiogenesis and growth of many axonal sprouts that increase the probability for axonal growth into the distal portion of the axonal tubes, ultimately inducing regeneration of the injured nerve. The ability of $\alpha$-gal nanoparticles to induce nerve regeneration may be studied by applying them within a conduit in which the nanoparticles are mixed with a semi-solid filler such as keratin hydrogelfilled conduit [42] or conduits containing nerve tissue ECM [43]. Additional possible fillers which may be used for applying $\alpha$-gal nanoparticles are described below.

\section{ADMINISTRATION OF $\alpha$-GAL NANOPARTICLES TO INTERNAL INJURIES BY PLASMA CLOT, FI- BRIN GLUE OR HYDROGEL}

Administration of $\alpha$-gal nanoparticles into internal spaces in the body requires the use of semi-solid media that prevent the diffusion of these nanoparticles throughout the body. Few examples of such media include plasma clots, fibrin glue and hydrogels. Since antibodies and complement proteins readily diffuse via these media, anti-Gal can reach the $\alpha$-gal nanoparticles, bind to them and activate the complement cascade in order to induce macrophage recruitment. An example of plasma cloth containing $\alpha$-gal nanoparticles and applied to wounds in the skin of GT-KO mice is described in Fig. (6). Plasma from human blood was mixed with $\alpha$-gal nanoparticles $(10 \mathrm{mg} / \mathrm{ml})$ and was induced to form a clot by 
addition of $10 \mathrm{mM}$ calcium chloride. Recruitment and infiltration of macrophages were observed within 3 days after placing the clot on the wound. However, after 6 days, the plasma clots were filled with macrophages recruited as a result of anti-Gal/ $\alpha$-gal nanoparticles interaction. The observed epidermal growth over the plasma clots may reflect the cytokines/growth factors secreted by the activated macrophages which stimulate regeneration of the epidermis [18]. It remains to be determined whether fillers such as hydrogels or fibrin glue containing $\alpha$-gal nanoparticles are also conducive to macrophage recruitment.

\section{INCORPORATION OF $\alpha$-GAL NANOPARTICLES INTO DECELLULARIZED TISSUE AND ORGAN IMPLANTS}

In the recent two decades, the research in generation of biodegradable natural biomaterials for tissue engineering has been extensive [44-46]. One exciting direction is the possible use of decellularized tissues and organs containing ECM that maintains the original scaffold architecture and composition. Conservation of the ECM in whole organs is being achieved by advanced dynamic decellularization techniques using perfusion of various detergents combined with DNA destroying solutions [44-46]. The ECM in decellularized tissues and organs instructs stem cells to differentiate into cells that restore the biological function of the injured tissue [4447]. Several studies have demonstrated the use of scaffold derived from decellularized porcine bladder submucosa for urethral tissue engineering [48], porcine decellularized myocardium for regeneration of the injured ventricular wall $[49,50]$ and porcine small intestinal submucosa for repair of small bowel tissue [51]. Because of the decellularization processing, these biomaterials are porous [45]. Thus, it is probable that by soaking them in an $\alpha$-gal nanoparticles suspension the nanoparticles will diffuse into such biomaterials.

When whole decellularized organs are used as biomaterials for tissue engineering, $\alpha$-gal nanoparticles penetration throughout the organ may be achieved by perfusion, similar to the perfusion of the decellularizing solutions into such organs [44-46]. Following implantation, these nanoparticles will bind anti-Gal since this antibody is ubiquitous in the body. The resulting complement activation will induce rapid migration of the macrophages into the implant as in Fig. (5). In order for these macrophages to induce regeneration of the implant, they have to differentiate into a pro-healing state $[52,53]$. This can be achieved by activation of these macrophages which bind anti-Gal coated $\alpha$-gal nanoparticles via the Fc/Fc $\gamma$ R interaction (Fig. 1A). The pro-healing activity of the activated macrophages may induce recruitment of stem cells that will be guided by the ECM of the implant to differentiate into the original cells that comprised the decellularized tissue. Thus, the $\alpha$-gal nanoparticles within the implant may shift the dynamics of cell repopulation from fibroblasts infiltration and fibrosis (as the default regenerative process) to the recruitment of stem cells that differentiate under the guidance of the ECM into the desired cells. In the case of a heart ventricular patch, this process may result in repopulation of the patch with cardiomyocytes that assist in the contractile activity of the ventricular myocardium, whereas in decellularized urinary bladder tissue, these recruitment and activation processes may result in the repopulation of the implant with smooth muscle cells and the covering of the surface with transitional epithelium.

In some natural biomaterials of nonprimate mammalian origin (e.g. of porcine origin), there may be the need to strip the implant of autologous $\alpha$-gal epitopes (e.g. by recombinant $\alpha$-galactosidase), or use implants from GT-KO pigs devoid of $\alpha$-gal epitopes. Since the $\alpha$-gal epitope is present both on cells and on glycoproteins of the ECM, binding of anti-Gal to these epitopes on the ECM may result in immune mediated destruction of the ECM. A study addressing this issue has been performed by implantation of ECM scaffold, prepared from porcine intestinal submucosa, in an anti-Gal producing monkey [54]. This study has indicated that despite presentation of $\alpha$-gal epitopes by this ECM, and the resulting elevation in anti-Gal titer, no adverse effects were observed upon tissue remodeling and regeneration of the connective tissue [54]. However, in decellularized organs containing epidermal components, ECM that includes basement membrane expresses much higher amounts of $\alpha$-gal epitopes, primarily because of the abundance of laminin that carries $50-70 \alpha$-gal epitopes per molecule and thus readily binds anti-Gal [55]. If anti-Gal binding to these multiple $\alpha$-gal epitopes interferes with the regeneration of the tissue, these epitopes can be removed by exposure of the ECM to recombinant $\alpha$-galactosidase (e.g. by perfusion, or immersion). The efficacy of porcine implants stripped of $\alpha$-gal epitopes in humans has been demonstrated in regeneration of anterior cruciate ligaments (ACL) in patients with torn ACL that were implanted with pig patellar-tibia tendon treated with recombinant $\alpha$-galactosidase and mildly cross-linked with glutaraldehyde [56].

\section{CONCLUSIONS}

$\alpha$-Gal nanoparticles accelerate healing of injuries by binding the natural anti-Gal antibody, activation of complement system for rapid recruitment of macrophages and activation of these macrophages into pro-healing macrophages. The cytokines secreted from these macrophages may recruit stem cells which are guided by the microenvironment to differentiate into various cells. It is possible that this treatment which accelerates healing of wounds may also be effective in repair and regeneration of internal injuries such as post infarction ischemic myocardium and injured nerves. $\alpha$-Gal nanoparticles may be administered to internal injuries within semi-solid fillers such as plasma clot, fibrin glue and hydrogels and may be incorporated into porous natural biomaterials for inducing the rapid in vivo repopulation of such implants with cells that may restore biological functions in the injured site.

\section{CONFLICT OF INTERESTS}

The author has no conflict of interests in the studies described in this review.

\section{ACKNOWLEDGEMENT}

Declared none.

\section{ABBREVIATIONS} $\alpha$-gal epitope $\quad=\quad \begin{aligned} & \text { Gal } \alpha 1-3 \text { Gal } \beta 1-4 G l c N A c-R \\ & \text { epitope }\end{aligned}$ 
$\alpha 1,3 \mathrm{GT}$

$=\alpha 1,3$ galactosyltransferase

$\alpha$-gal nanoparticles $=$ submicroscopic liposomes with glycolipids that carry multiple $\alpha$-gal epitopes

GT- KO mice or pigs $\quad=\quad$ knockout mice or pigs for the $\alpha 1,3$ galactosyltransferase gene

RBC $\quad=\quad$ red blood cells

\section{REFERENCES}

[1] Martin P. Wound Healing- Aiming for Perfect Skin Regeneration. Science $1997 ; 276: 75-81$

[2] Singer AJ, Clark RA. Cutaneous wound healing. N Engl J Med 1997; 341: 738-46.

[3] Heinrich SA, Messingham KA, Gregory MS, et al. Elevated monocyte chemoattractant protein-1 levels following thermal injury precede monocyte recruitment to the wound site and are controlled, in part, by tumor necrosis factor- $\alpha$. Wound Repair Regen 2003; 11 : 110-9.

[4] Low QE, Drugea IA, Duffner LA, et al. Wound healing in MIP$1 \alpha(-/-)$ and MCP-1(-/-) mice. Am J Pathol 2001; 159: 457-63.

[5] Snyderman R, Pike MC. Chemoattractant receptors on phagocytic cells. Annu Rev Immunol 1984; 2: 257-81.

[6] Haeney MR. The role of the complement cascade in sepsis. J Antimicrobial Chemother 1998; 41: 41-6.

[7] Galili U, Rachmilewitz EA, Peleg A, Flechner I. A unique natural human IgG antibody with anti- $\alpha$-galactosyl specificity. J Exp Med 1984; 160: 1519-31.

[8] Wang L, Anaraki F, Henion TR, Galili U. Variations in activity of the human natural anti-Gal antibody in young and elderly populations. J Gerontol (Med Sci) 1995; 50A: M227-33.

[9] Galili U, Macher BA, Buehler J, Shohet SB. Human natural anti- $\alpha$ galactosyl IgG. II. The specific recognition of $\alpha(1-3)$-linked galactose residues. J Exp Med 1985; 162: 573-82.

[10] Galili U, Mandrell RE, Hamadeh RM, Shohet SB, Griffis JM. Interaction between human natural anti- $\alpha$-galactosyl immunoglobulin $\mathrm{G}$ and bacteria of the human flora. Infect Immun 1988; 56: 1730-7.

[11] Galili U, Clark MR, Shohet SB, Buehler J, Macher BA. Evolutionary relationship between the anti-Gal antibody and the Gal $1 \rightarrow 3$ Gal epitope in primates. Proc Natl Acad Sci USA 1987; 84:1369-73.

[12] Galili U, Shohet SB, Kobrin E, Stults CLM, Macher BA. Man, apes, and Old World monkeys differ from other mammals in the expression of $\alpha$-galactosyl epitopes on nucleated cells. J Biol Chem $1988 ; 263: 17755-62$.

[13] Galili U, Swanson K. Gene sequences suggest inactivation of $\alpha 1-3$ galactosyltransferase in catarrhines after the divergence of apes from monkeys. Proc Natl Acad Sci USA 1991; 88: 7401-4.

[14] Galili U, Andrews P. Suppression of $\alpha$-galactosyl epitopes synthesis and production of the natural anti-Gal antibody: A major evolutionary event in ancestral Old World primates. J Hum Evol 1995; 29: 433-42.

[15] Larsen RD, Rivera-Marrero CA, Ernst LK, Cummings RD, Lowe JB. Frameshift and nonsense mutations in a human genomic sequence homologous to a murine UDP-Galß-D-Gal(1,4)-DGlcNAc $\alpha(1,3)$ galactosyltransferase cDNA. J Biol Chem 1990; 265: 7055-61.

[16] Joziasse DH, Shaper JH, Jabs EW, Shaper NL. Characterization of an $\alpha 1$-3-galactosyltransferase homologue on human chromosome 12 that is organized as a processed pseudogene. J Biol Chem 1991; 266: 6991-8.

[17] Koike C, Uddin M, Wildman DE, et al. Functionally important glycosyltransferase gain and loss during catarrhine primate emergence. Proc Natl Acad Sci USA 2007; 104: 559-64.

[18] Wigglesworth KM, Racki WJ, Mishra R, et al. Rapid recruitment and activation of macrophages by anti-Gal/ $\alpha$-gal liposome interaction accelerates wound healing. J Immunol 2011; 186: 4422-32.

[19] Hurwitz Z, Ignotz R, Lalikos J, Galili U. Accelerated porcine wound healing with $\alpha$-Gal nanoparticles. Plast Reconstr Surg 2012; 129: $242-51$.
[20] Eto T, Iichikawa Y, Nishimura K, Ando S, Yamakawa T. Chemistry of lipids of the posthemolytic residue or stroma of erythrocytes. XVI. Occurance of ceramide pentasaccharide in the membrane of erythrocytes and reticulocytes in rabbit. J Biochem (Tokyo) 1968; 64: 205-13.

[21] Stellner K, Saito H, Hakomori S. Determination of aminosugar linkage in glycolipids by methylation. Aminosugar linkage of ceramide pentasaccharides of rabbit erythrocytes and of Forssman antigen. Arch Biochem Biophys 1973; 133: 464-72.

[22] Dabrowski U, Hanfland P, Egge H, Kuhn S, Dabrowski J. Immunochemistry of $\mathrm{I} / \mathrm{i}$-active oligo- and polyglycosylceramides from rabbit erythrocyte membranes. Determination of branching patterns of a ceramide pentadecasaccharide by $1 \mathrm{H}$ nuclear magnetic resonance. J Biol Chem 1984; 259: 7648-51.

[23] Egge H, Kordowicz M, Peter-Katalinic J, Hanfland P. Immunochemistry of I/i-active oligo- and polyglycosylceramides from rabbit erythrocyte membranes. J Biol Chem 1985; 260: 4927-35.

[24] Galili U, Wigglesworth K, Abdel-Motal UM. Accelerated healing of skin burns by anti-Gal/ $\alpha$-gal liposomes interaction. Burns 2010; 36: $239-51$.

[25] Galili U, Wigglesworth K, Abdel-Motal UM. Intratumoral injection of $\alpha$-gal glycolipids induces xenograft-like destruction and conversion of lesions into endogenous vaccines. J Immunol 2007; 178: 4676-87.

[26] Thall AD, Maly P, Lowe JB. Oocyte Gal $\alpha 1,3 \mathrm{Gal}$ epitopes implicated in sperm adhesion to the zona pellucida glycoprotein $\mathrm{ZP} 3$ are not required for fertilization in the mouse. J Biol Chem 1995; 270: 21437-40.

[27] Tearle RG, Tange MJ, Zannettino ZL, et al. The $\alpha-1,3-$ galactosyltransferase knockout mouse. Implications for xenotransplantation. Transplantation 1996; 61: 13-9.

[28] Lai L, Kolber-Simonds D, Park KW, et al. Production of $\alpha-1,3-$ galactosyltransferase knockout pigs by nuclear transfer cloning. Science 2002; 295: 1089-92.

[29] Phelps CJ, Koike C, Vaught TD, et al. Production of $\alpha 1,3-$ galactosyltransferase-deficient pigs. Science 2003; 299: 411-4.

[30] Kolber-Simonds D, Lai L, Watt SR, et al. Production of $\alpha-1,3-$ galactosyltransferase null pigs by means of nuclear transfer with fibroblasts bearing loss of heterozygosity mutations. Proc Natl Acad Sci USA 2004; 101: 7335-40.

[31] Simon PM, Neethling FA, Taniguchi S, et al. Intravenous infusion of Gala1-3Gal oligosaccharides in baboon delays hyperacute rejection of porcine heart xenografts. Transplantation 1998; 56: 346-53.

[32] Xu Y, Lorf T, Sablinski T, et al. Removal of anti-porcine natural antibodies from human and nonhuman primate plasma in vitro and in vivo by a Gal $\alpha 1-3 \mathrm{Gal} \beta 1-4 \beta-G l c-X$ immunoaffinity column. Transplantation 1998; 65: 172-9.

[33] Collins BH, Cotterell AH, McCurry KR, et al. Cardiac xenografts between primate species provide evidence of the $\alpha$-galactosyl determinant in hyperacute rejection. J Immunol 1995; 154: 5500-10.

[34] Shilo S, Roth S, Amzel T, et al. Cutaneous wound healing after treatment with plant-derived human recombinant collagen flowable gel. Tissue Eng Part A. 2013; 19(13-14): 1519-26.

[35] Minatoguchi S, Takemura G, Chen XH, et al. Acceleration of the healing process and myocardial regeneration may be important as a mechanism of improvement of cardiac function and remodeling by postinfarction granulocyte colony-stimulating factor treatment. Circulation 2004; 109: 2572-80.

[36] Dewald O, Zymek P, Winkelmann K, et al. CCL2/Monocyte Chemoattractant Protein-1 regulates inflammatory responses critical to healing myocardial infarcts. Circ Res 2005; 96: 881-9.

[37] Yano T, Miura T, Whittaker P, Miki T, et al. Macrophage colonystimulating factor treatment after myocardial infarction attenuates left ventricular dysfunction by accelerating infarct repair. J Am Coll Cardiol 2006; 47: 626-34.

[38] Strauer BE, Brehm M, Zeus T, et al. Repair of infarcted myocardium by autologous intracoronary mononuclear bone marrow cell transplantation in humans. Circulation 2002; 106: 1913-8.

[39] van Amerongen MJ, Harmsen MC, van Rooijen N, Petersen AH, van Luyn MJ. Macrophage depletion impairs wound healing and increases left ventricular remodeling after myocardial injury in mice. Am J Pathol 2007; 170: 818-29.

[40] Dray C, Rougon G, Debarbieux F. Qunatitative analysis by in vivo imaging of the dynamics of vascular and axonal networks in injured mouse spinal cord. Proc Natl Acad Sci USA 2009; 106: 945964. 
[41] Mokarram N, Merchant A, Mukhatyar V, Patel G, Bellamkonda $\mathrm{RV}$. Effect of modulating macrophage phenotype on peripheral nerve repair. Biomaterials 2012; 33: 8793-801

[42] Apel PJ, Garrett JP, Sierpinski P, et al. Peripheral nerve regeneration using a keratin-based scaffold: long-term functional and histological outcomes in a mouse model. J Hand Surg Am 2008; 33: 1541-7.

[43] Crapo PM, Medberry CJ, Reing JE, et al. Biologic scaffolds composed of central nervous system extracellular matrix. Biomaterials 2012; 33: 3539-47.

[44] Atala A. Engineering organs. Curr Opin Biotechnol 2009; 20: 57593.

[45] Crapo PM, Gilbert TW, Badylak SF. An overview of tissue and whole organ decellularization processes. Biomaterials 2011; 32: 3233-43.

[46] Badylak SF, Weiss DJ, Caplan A, Macchiarini P. Engineered whole organs and complex tissues. Lancet 2012; 379: 943-52.

[47] Arenas-Herrera JE, Ko IK, Atala A, Yoo JJ. Decellularization for whole organ bioengineering. Biomed Mater 2013; 8(1): 014106.

[48] Liu Y, Bharadwaj S, Lee SJ, Atala A, Zhang Y. Optimization of a natural collagen scaffold to aid cell-matrix penetration for urologic tissue engineering. Biomaterials 2009; 30: 3865-73.

[49] Horton RE, Auguste DT. Synergistic effects of hypoxia and extracellular matrix cues in cardiomyogenesis. Biomaterials 2012; 33: 6313-9.
[50] Sarig U, Au-Yeung GC, Wang Y, et al. Thick acellular heart extracellular matrix with inherent vasculature: a potential platform for myocardial tissue regeneration. Tissue Eng Part A 2012; 18: 212537.

[51] Chen MK, Badylak SF. Small bowel tissue engineering using small intestinal submucosa as scaffold. J Surg Res 2001; 99: 352-8.

[52] Zhang L, Cao Z, Bai T, et al. Zwitterionic hydrogels implanted in mice resist the foreign-body reaction. Nat Biotechnol 2013; 31: 553-6.

[53] Tian H, Lu Y, Shah SP, Hong S. Autacoid 14S,21R-dihydroxydocosahexaenoic acid counteracts diabetic impairment of macrophage prohealing functions. Am J Pathol 2011; 179: 1780-91.

[54] Daly KA, Stewart-Akers AM, Hara H, et al. Effect of the $\alpha-G a l$ epitope on the response to small intestinal submucosa extracellular matrix in a nonhuman primate model. Tissue Eng. Part A. 2009; 15: 3877-88.

[55] Galili, U. Evolution and pathophysiology of the human natural anti-Gal antibody. Springer Semin Immunopathol 1993; 15: 15571.

[56] Stone KR, Abdel-Motal UM, Walgenbach AW, Turek TJ, Galili U. Replacement of human anterior cruciate ligaments with pig ligaments: a model for anti-non-gal antibody response in long-term xenotransplantation. Transplantation 2007; 83: 211-9.

[57] Galili U. Discovery of the natural anti-Gal antibody and its past and future relevance to medicine. Xenotransplantation 2013; 20: 13847.

Received: April 13, 2013

Revised: June 03, 2013

Accepted: June 03, 2013

(C) Uri Galili; Licensee Bentham Open.

This is an open access article licensed under the terms of the Creative Commons Attribution Non-Commercial License (http: //creativecommons.org/licenses/by$\mathrm{nc} / 3.0 /$ ), which permits unrestricted, non-commercial use, distribution and reproduction in any medium, provided the work is properly cited. 